\title{
Textbook of Global Mental Health: Trauma and Recovery. A companion guide for field and clinical care of traumatized people worldwide
}

\author{
Harvard Program in Refugee Trauma (2012), ISBN 781105851087 \\ Editor: Richard F. Mollica
}

Inke Schaap, MSc, RN*

The major aim of this book is to aid the work of those professionals struggling daily at the local level to restore the health and wellbeing of the millions of people whose lives are disrupted each year. Mass violence and disaster create a new historical space where actions and behaviours that once seemed incomprehensible in daily life emerge as normal, Mollica claims. The aid that was given internationally in the past took advantage of the accumulating scientific knowledge derived from decades of fieldwork caring for traumatized persons. In this textbook, an overview of this knowledge is provided. It grew out of an historic meeting of the world's Ministers of Health in Rome in 2004 (called Project 1 Billion for the number of people affected by violence) to establish and disseminate the first Global Mental Health (GMH) Action Plan. This plan offers a culturally valid, science based guide to the development and implementa-

\footnotetext{
^) Saxion University

Correspondence to: inkeschaap@yahoo.com
}

tion of ongoing mental health programmes in post conflict areas. The complete text of the Global Mental Health Action Plan is part of the book.

The eight dimensions of the plan are chapters in this book. Each chapter contains a state-of-the-art knowledge from science and culture and is written by experts on politics, mental health, economic development, humanitarian aid and human rights. The chapters are not intended to stand-alone but are part of an integrated approach of trauma and recovery. It is one of the aims of this book to 'break down the silos that often define health/mental health activities' (p.40). Mollica illuminates this with examples of the mental health professional who reveals a complete lack of knowledge of financing and human rights workers who have little knowledge of clinical areas of expertise.

The eight dimensions of the GMH Action Plan are as follows: mental health policy and legislation, financing of mental health recovery, science-based mental health services, building an ongoing programme of mental health education, coordination of international agencies, mental health linkages to economic development, mental health and 
human rights, evaluation, research and ethics. Every dimension is a section in this textbook.

In the clear and complete introduction, Mollica describes the background and the practical implications of the GMH Action Plan. In a table, four steps are presented for analysis of a clinical case according to the Plan. After the description of the problem, the desired results need to be stated simply. The next step is to review all eight dimensions of the GMH Action Plan by assessing each of their strengths and barriers. This analysis will be used in the final step to plan and act on a strategy to achieve the desired results by transforming all eight dimensions. These steps are made clear by two cases. The first is the case of Liberia in which a strategy to implement the GMH action plan is developed. The second case leads to a plan of care for a woman with acute stress and drug abuse and several social problems who flew from Cambodia to the U.S.

The book contains an accessible overview of practical facts and measures. It becomes clear from the Global Mental Health Survey that is held in 17 post-conflict countries that the Action Plan is necessary; the mental health care in a many countries is absent or the quality is below standards. Bosnia and Herzegovina are exceptions; the development of mental health care in those countries is widely acknowledged. The essay by Freeman that follows this chapter on policymaking is inspiring and justifies the pragmatic considerations that need to be made in daily practice. Measuring burden of disease is one of the topics in the section on financing. The author proposes to measure Gross National Happiness instead of Gross National Product. The needs of post conflict countries are described and it is concluded that regarding these needs, ongoing mental health programmes are necessary.
With regards to science based mental health services, the importance of a community approach is emphasized. With this approach, not only mental health (services) will be restored but also social conditions. In the next chapter, the identification of, and interventions with, victims of torture are described. The mental health lessons learned from the Lebanon war studies are also interesting. There is a good overview of the consequences of mass violence and trauma for children and adolescents. Psychosocial support in schools is one of the interventions that is highlighted. In this section of the book, there is also a chapter on the role of religion and spirituality. The last chapter on burnout and professionals makes this section a complete and clear overview.

In 'Building an ongoing programme of mental health education' ways of education (online teaching programmes) are described as well as the process of learning. By means of Communities of Practice, learning is a way to promote social cohesion in a society. In this section, the Balint Groups are mentioned as emotional education.

In 'Coordination of international agencies' a conceptual framework for psychosocial interventions is presented. In the humanitarian approach of violence the triangle of aggressor, victim, rescuer is described. This approach gets an extra dimension by adding the role of media and the influences of social and mental health theories. In this chapter, the importance of resilience is also stressed.

In the section on mental health linkages to economic development, the costs and consequences of bad mental health are described. It is of great importance that those who are responsible for the planning of the recovery of society are aware of these facts.

The seventh dimension of the Global Mental Health Action Plan consists of 
Mental health and human rights. This chapter gives an overview of legislation and instruments. It is stressed that there is a correlation between mental health recovery and reconciliation. The psychological treatment of trauma cannot be seen without acknowledgement of political aspects.

In the concluding section on evaluation, research and ethics, innovative ways of evaluation of mental health (services) in post conflict countries are proposed, such as process-evaluation needs assessment or patient satisfaction. There is an overview of the most widely used instruments to measure (the impact of) trauma and torture. In the last chapter of the textbook, ethics of research is discussed. The vulnerable position of victims is stressed and therefore researchers should be even more attentive to carry out the best research governance possible.

The 'textbook of global mental health: trauma and recovery' looks huge and it is huge in its content. It is a must read for professionals in the field of trauma and recovery and for professionals who are interested in this field. 